Internat. J. Math. \& Math. Sci.

Vol. 24, No. 8 (2000) 533-538

S0161171200003732

(C) Hindawi Publishing Corp.

\title{
MATRIX TRANSFORMATIONS FROM ABSOLUTELY CONVERGENT SERIES TO CONVERGENT SEQUENCES AS GENERAL WEIGHTED MEAN SUMMABILITY METHODS
}

\author{
JINLU LI \\ (Received 10 May 1999 and in revised form 22 July 1999)
}

ABSTRACT. We prove the necessary and sufficient conditions for an infinity matrix to be a mapping, from absolutely convergent series to convergent sequences, which is treated as general weighted mean summability methods. The results include a classical result by Hardy and another by Moricz and Rhoades as particular cases.

Keywords and phrases. Weighted mean matrix, matrix transformation.

2000 Mathematics Subject Classification. Primary 40C10, 40D25, 40G05.

1. Introduction. A series

$$
\sum_{k=0}^{\infty} x_{k}
$$

of complex numbers is said to be summable $(C, 1)$ if the sequence

$$
\frac{1}{n+1} \sum_{k=0}^{n} \sum_{i=0}^{k} x_{i}, \quad n=0,1,2, \ldots
$$

converges to a finite limit as $n \rightarrow \infty$.

In [1] Hardy proved the following theorem.

THEOREM 1.1. The series (1.1) is summable $(C, 1)$ to a finite number $L$ if and only if the series

$$
\sum_{n=0}^{\infty} \sum_{k=n}^{\infty} \frac{x_{k}}{k+1}
$$

converges to the same limit.

For a sequence of positive numbers $\left(p_{n}\right)$, let $P_{n}:=\sum_{k=0}^{n} p_{n}$. A weighted mean matrix $\bar{N}$ is an infinity lower triangular matrix with entries (see [2])

$$
a_{n k}:=\frac{p_{k}}{P_{n}}, \quad k=0,1,2, \ldots, n, n=0,1,2, \ldots .
$$

The series (1.1) is said to be summable $\bar{N}$ if the following sequence:

$$
\frac{1}{P_{n}} \sum_{k=0}^{n} p_{k} \sum_{i=0}^{k} x_{i}, \quad n=0,1,2, \ldots,
$$

converges to a finite limit as $n \rightarrow \infty$. 
It is clear that summable $(C, 1)$ is a special case of summable $\bar{N}$, where

$$
p_{k}=1, \quad k=0,1,2, \ldots
$$

Based on the above idea, Moricz and Rhoades [2] established a result for a broad class of summability methods, which include the method of summability $(C, 1)$ as a particular case.

THEOREM 1.2. Let $\bar{N}$ be a weighted mean matrix determined by a sequence $\left(p_{n}\right)$ of positive numbers such that the following conditions are satisfied:

$$
\begin{gathered}
P_{n} \rightarrow \infty, \quad \frac{p_{n}}{P_{n}} \longrightarrow 0 \text { as } n \rightarrow \infty, \\
\sup _{n \geq 0}\left\{\frac{p_{n+1} p_{n-1}}{p_{n} P_{n+1}}+P_{n} \sum_{k=n}^{\infty} \frac{1}{P_{n+1}}\left|\frac{p_{k+1}}{p_{k}}-\frac{p_{k+2} P_{k}}{p_{k+1} P_{k+2}}\right|\right\}<\infty, \\
\sup _{n \geq 0}\left\{\frac{p_{n}}{p_{n+1}}+\frac{1}{P_{n}} \sum_{k=0}^{n}\left|\frac{p_{k} P_{k+1}}{p_{k+1}}-\frac{p_{k-1} P_{k-1}}{p_{k}}\right|\right\}<\infty,
\end{gathered}
$$

with the agreement that

$$
p_{-1}=P_{-1}:=0
$$

Then the series (1.1) is summable $\bar{N}$ to a finite number $L$ if and only if the series

$$
\sum_{n=0}^{\infty} \sum_{k=n}^{\infty} \frac{p_{n}}{P_{k}} x_{k}
$$

converges to the same limit $L$.

In this paper, we will study the matrix transformations from the space of absolutely convergent series of complex numbers, $l_{1}$, to the space of convergent sequences of complex numbers, $c$. Then we shall establish a more general result for a broader class of weighted mean methods, which includes the method of summable $\bar{N}$ as a particular case if the series (1.1) is absolutely convergent.

2. Matrix transformations from $l_{1}$ to $c$. Let $A=\left(a_{n k}\right)$ be an infinity matrix with complex entries and let $l$ denote the linear space of complex number sequences. For a sequence $x=\left(x_{n}\right) \in l, A x$ is in $l$ and its entries are given by

$$
(A x)_{n}=\sum_{k=0}^{\infty} a_{n k} x_{k}, \quad n=0,1,2, \ldots
$$

provided the series converges to a finite complex number.

The following result is well known (see [3, 4]); we list it as a proposition.

Proposition 2.1. Let $a=\left(a_{k}\right)$ be a sequence of complex numbers. If for every $x=\left(x_{n}\right) \in l_{1}$, the series

$$
\sum_{k=0}^{\infty} a_{k} x_{k}
$$


converges to a finite complex number, then

$$
\sup _{k \geq 0}\left\{\left|a_{k}\right|\right\}<\infty .
$$

From Proposition 2.1, we have the following interesting result.

Proposition 2.2. Let $a=\left(a_{k}\right)$ be a sequence of complex numbers. If for every $x=\left(x_{n}\right) \in l_{1}$, the series

$$
\sum_{k=0}^{\infty} a_{k} x_{k}
$$

converges to a finite complex number, then the linear functional $f_{a}$ defined on $l_{1}$ by

$$
f_{a}(x)=\sum_{k=0}^{\infty} a_{k} x_{k}
$$

is a continuous (bounded) linear functional on $l_{1}$, such that

$$
\left\|f_{a}\right\|=\sup _{k \geq 0}\left\{\left|a_{k}\right|\right\} .
$$

From Proposition 2.1, we know that $A$ is well defined as a mapping from $l_{1}$ to $l$, if and only if

$$
\sup _{k \geq 0}\left\{\left|a_{n k}\right|\right\}<\infty, \quad \text { for } n=0,1,2, \ldots .
$$

The following result has been proved in [4] by using functional analysis techniques. It is also proved by summability methods. We list the following theorem without proof.

THEOREM 2.3. Let $A=\left(a_{n k}\right)$ be an infinity matrix with complex entries. Then $A$ is a mapping from $l_{1}$ to $c$, if and only if the following conditions are satisfied:

(i) for every fixed $k=0,1,2, \ldots$, the sequence $\left(a_{n k}\right)$ converges to a finite limit as $n \rightarrow \infty$,

(ii) $\sup _{n, k \geq 0}\left\{\left|a_{n k}\right|\right\}<\infty$.

Furthermore, if $A=\left(a_{n k}\right)$ satisfies conditions (i) and (ii), then for every $x=\left(x_{n}\right) \in l_{1}$, we have

$$
\lim _{n \rightarrow \infty}(A x)_{n}=\lim _{n \rightarrow \infty} \sum_{k=0}^{\infty} a_{n k} x_{k}=\sum_{k=0}^{\infty}\left(\lim _{n \rightarrow \infty} a_{n k}\right) x_{k} .
$$

The following corollary follows from Theorem 2.3 and (2.8).

COROLLARY 2.4. Let $A=\left(a_{n k}\right)$ be an infinity matrix with complex entries. If $A$ is a mapping from $l_{1}$ to $c$, then the linear operator $A$ is continuous (bounded) linear operator such that

$$
\|A\|=\sup _{n, k \geq 0}\left\{\left|a_{n k}\right|\right\} .
$$


3. Applications to summable $(C, 1)$ and summable $\bar{N}$. The following corollary comes immediately from Theorem 2.3 , which describes an equivalent reformulation of summability by more general weighted mean methods which are matrix transformations.

COROllary 3.1. Let $A=\left(a_{n k}\right), B=\left(b_{n k}\right)$ be two infinity matrices with complex entries. Suppose $A, B$ are mapping from $l_{1}$ to $c$, that is $A, B$ satisfying conditions (i), (ii) of Theorem 2.3. Then for every $x=\left(x_{n}\right) \in l_{1}$,

$$
\lim _{n \rightarrow \infty}(A x)_{n}=\lim _{n \rightarrow \infty}(B x)_{n}
$$

if and only if for every fixed $k=0,1,2, \ldots$,

$$
\lim _{n \rightarrow \infty} a_{n k}=\lim _{n \rightarrow \infty} b_{n k}
$$

Proof. Since $A, B$ satisfy conditions (i), (ii) of Theorem 2.3, then from (2.8), we have

$$
\begin{aligned}
& \lim _{n \rightarrow \infty}(A x)_{n}=\lim _{n \rightarrow \infty} \sum_{k=0}^{\infty} a_{n k} x_{k}=\sum_{k=0}^{\infty}\left(\lim _{n \rightarrow \infty} a_{n k}\right) x_{k}, \\
& \lim _{n \rightarrow \infty}(B x)_{n}=\lim _{n \rightarrow \infty} \sum_{k=0}^{\infty} b_{n k} x_{k}=\sum_{k=0}^{\infty}\left(\lim _{n \rightarrow \infty} b_{n k}\right) x_{k},
\end{aligned}
$$

for any $x=\left(x_{n}\right) \in l_{1}$. From (2.8) and (3.4), we see that (3.2) implies (3.1). Now, for every fixed $k=0,1,2, \ldots$, define $x=\left(x_{i}\right)$ by

$$
x_{i}= \begin{cases}1, & \text { if } i=k, \\ 0, & \text { if } i \neq k\end{cases}
$$

It is clear that $x \in l_{1}$. Equations (2.8) and (3.4) imply

$$
\lim _{n \rightarrow \infty}(A x)_{n}=\lim _{n \rightarrow \infty} a_{n k}, \quad \lim _{n \rightarrow \infty}(B x)_{n}=\lim _{n \rightarrow \infty} b_{n k} .
$$

From (3.6), we see that (3.1) implies (3.2).

Recall that for a sequence of positive numbers $\left(p_{n}\right), P_{n}=\sum_{k=0}^{n} p_{k}$. The series (1.1) is said to be summable $\bar{N}$ if the following sequence:

$$
\frac{1}{P_{n}} \sum_{k=n}^{n} p_{k} \sum_{i=0}^{k} x_{i}, \quad n=0,1,2, \ldots
$$

converges to a finite limit as $n \rightarrow \infty$.

To generalize Theorem 1.2, we shall construct two weighted mean matrices according to the summability (3.7) and the following summability method:

$$
\sum_{n=0}^{\infty} \sum_{k=n}^{\infty} \frac{p_{n}}{P_{k}} x_{k} .
$$


Based on the sequence of positive numbers $\left(p_{n}\right)$, define two infinity matrices $A=$ $\left(a_{n k}\right)$ and $B=\left(b_{n k}\right)$, by

$$
\begin{aligned}
& a_{n k}= \begin{cases}0, & \text { if } k>n, \\
\frac{P_{n}-P_{k-1}}{P_{n}}, & \text { if } k \leq n,\end{cases} \\
& b_{n k}= \begin{cases}\frac{P_{n}}{P_{k}}, & \text { if } k>n, \\
1, & \text { if } k \leq n,\end{cases}
\end{aligned}
$$

where we agree that $P_{-1}=0$.

It can be seen that any sequence of positive numbers $\left(p_{n}\right), B=\left(b_{n k}\right)$ defined by (3.10), always satisfies the conditions (i) and (ii) of Theorem 2.3 and $A=\left(a_{n k}\right)$ defined by (3.9) always satisfies the conditions (ii) of Theorem 2.3. Furthermore, $A=\left(a_{n k}\right)$ will satisfies the conditions (i) of Theorem 2.3 if the sequence $\left(p_{n}\right)$ satisfies

$$
P_{n} \rightarrow \infty \quad \text { as } n \rightarrow \infty \text {. }
$$

Hence we have the following corollary of Theorem 2.3.

COROLlaRY 3.2. For any sequence of positive numbers $\left(p_{n}\right), B=\left(b_{n k}\right)$ defined by (3.10) is always a mapping from $l_{1}$ to c. If $\left(p_{n}\right)$ satisfying (3.11), then $A=\left(a_{n k}\right)$ defined by (3.9) is a mapping from $l_{1}$ to $c$.

The following corollary will give the Moricz and Rhoades's result, Theorem 1.2, if the series (1.1) is absolutely convergent.

COROLLARY 3.3. Let $\left(p_{n}\right)$ be a sequence of positive numbers satisfying (3.11). Let $A=\left(a_{n k}\right), B=\left(b_{n k}\right)$ be defined by (3.9) and (3.10). Then for any $x=\left(x_{n}\right) \in l_{1}$, we have

$$
\lim _{n \rightarrow \infty}(A x)_{n}=\lim _{n \rightarrow \infty}(B x)_{n}=\sum_{k=0}^{\infty} x_{k} .
$$

Proof. Notice that under condition (3.11), we have that for every fixed $k=0,1,2, \ldots$,

$$
\lim _{n \rightarrow \infty} a_{n k}=\lim _{n \rightarrow \infty} b_{n k}=1 \text {. }
$$

Then the proof of this corollary follows Corollary 3.2 and the equalities (2.8) and (3.4) immediately.

From the definitions (3.9) and (3.10), we see that for every fixed $n=0,1,2, \ldots$,

$$
(A x)_{n}=\frac{1}{P_{n}} \sum_{k=0}^{n} p_{k} \sum_{i=0}^{k} x_{i}, \quad(B x)_{n}=\sum_{m=0}^{n} \sum_{k=m}^{\infty} \frac{p_{n}}{P_{k}} x_{k} .
$$

Corollary 3.3 shows that if the sequence of positive numbers $\left(p_{n}\right)$ satisfies condition (3.11), then for any $x=\left(x_{n}\right) \in l_{1}$, we have

$$
\lim _{n \rightarrow \infty} \frac{1}{P_{n}} \sum_{k=0}^{n} p_{k} \sum_{i=0}^{k} x_{i}=\sum_{n=0}^{\infty} \sum_{k=n}^{\infty} \frac{p_{n}}{P_{k}} x_{k}=\sum_{k=0}^{\infty} x_{k} .
$$


In a particular case, as mentioned by Moricz and Rhoades [2], taking $p_{k}=1$, for $k=$ $0,1,2, \ldots$, we find the Hardy's result, Theorem 1.1, if that the series (1.1) is absolutely convergent, that is, for any $x=\left(x_{n}\right) \in l_{1}$,

$$
\lim _{n \rightarrow \infty} \frac{1}{n+1} \sum_{k=0}^{n} \sum_{i=0}^{k} x_{i}=\sum_{n=0}^{\infty} \sum_{k=n}^{\infty} \frac{x_{k}}{k+1}=\sum_{k=0}^{\infty} x_{k} .
$$

Acknowledgements. The author wishes to thank Prof. J. Fridy and Prof. B. E. Rhoades for their communication and encouragement. The author would like to thank the referee for valuable suggestions which improved the presentation of the paper.

\section{REFERENCES}

[1] G. H. Hardy, A theorem concerning summable series, Pro. Cambridge Philos. Soc. 20 (19201921), 304-307.

[2] F. Móricz and B. E. Rhoades, An equivalent reformulation of summability by weighted mean methods, Linear Algebra Appl. 268 (1998), 171-181. MR 99c:40001. Zbl 897.40003.

[3] M. Stieglitz and H. Tietz, Matrixtransformationen von Folgenräumen. Eine Ergebnisübersicht, Math. Z. 154 (1977), no. 1, 1-16. MR 56\#5109. Zbl 345.40004.

[4] A. Wilansky, Summability through Functional Analysis, North-Holland Mathematics Studies, 85, North-Holland Publishing Co., Amsterdam, New York, Oxford, 1984, Notas de Matemática [Mathematical Notes], 91. MR 85d:40006. Zbl 531.40008.

Jinlu li: Department of Mathematics, Shawnee State University, Portsmouth, Ohio 45662, USA 


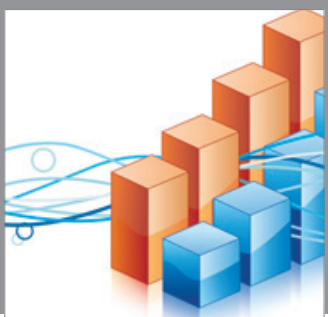

Advances in

Operations Research

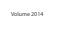

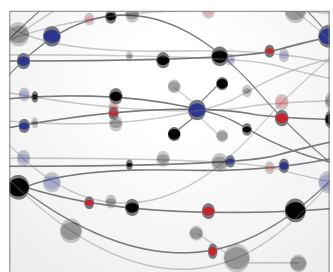

\section{The Scientific} World Journal
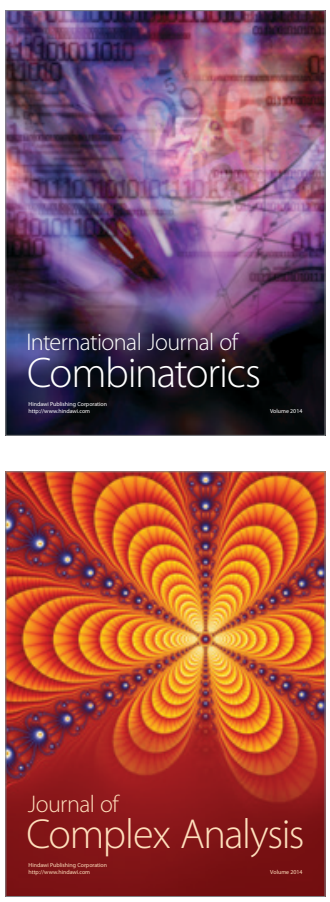

International Journal of

Mathematics and

Mathematical

Sciences
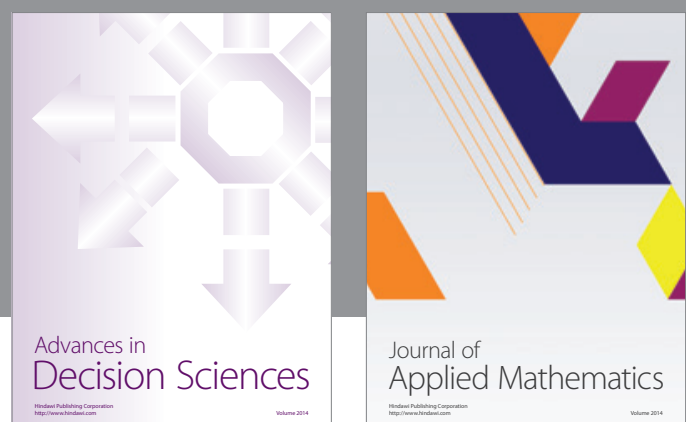

Journal of

Applied Mathematics
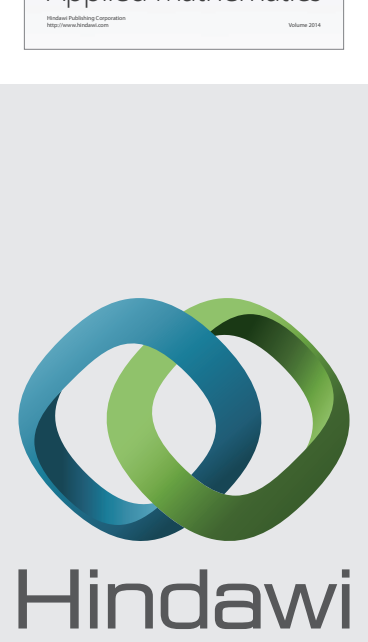

Submit your manuscripts at http://www.hindawi.com
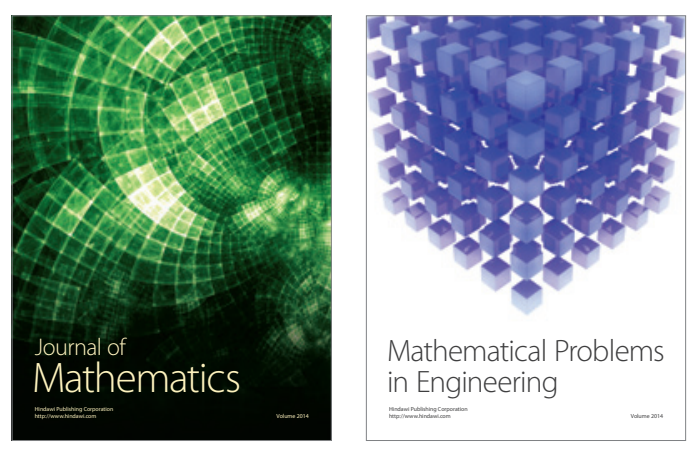

Mathematical Problems in Engineering
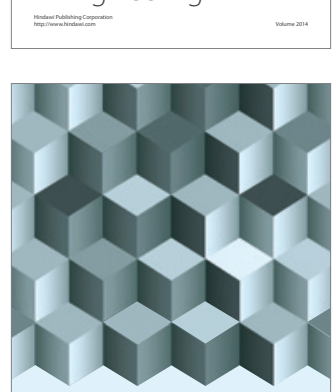

Journal of

Function Spaces
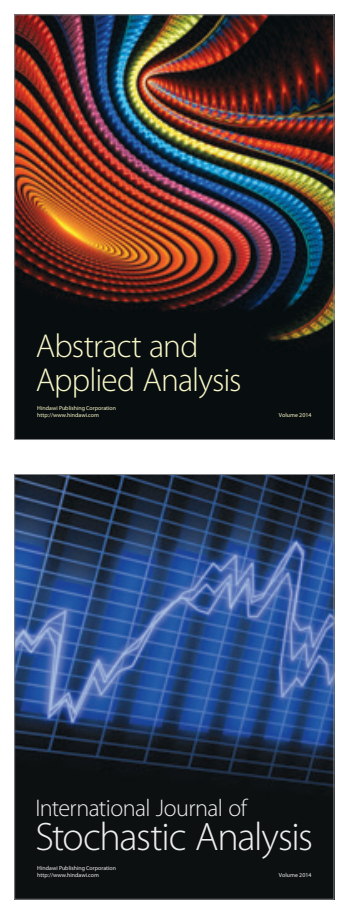

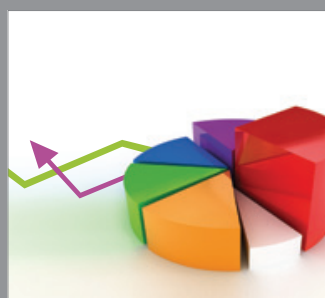

ournal of

Probability and Statistics

Promensencen
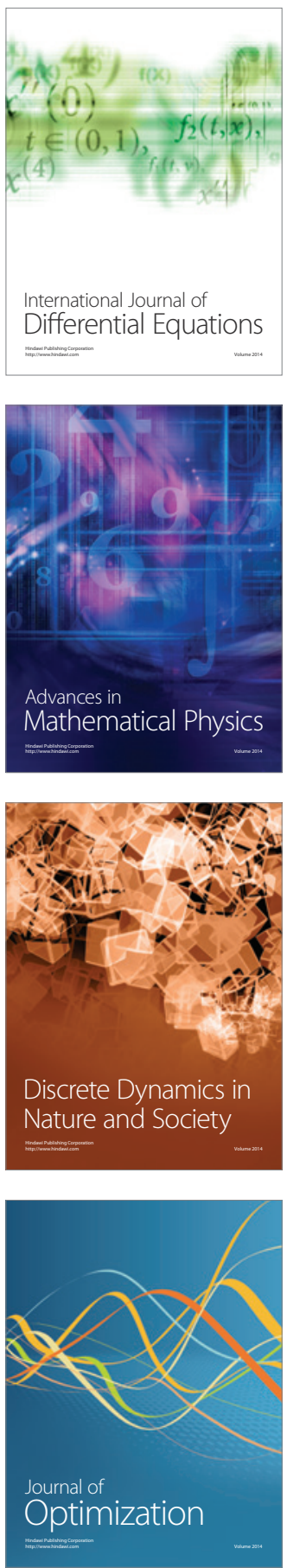